\title{
Modelo de Precificação de Capital: Segmento de Commodities Agrícola
}

\author{
Capital Asset Pricing Model: Sector of Agricultural Commodities
}

Tácito Augusto Farias ${ }^{1}$

Luiz Eduardo Nascimento Figueiredo ${ }^{2}$

Fábio Rodrigues Moura ${ }^{3}$

Resumo: O objetivo do artigo é a aplicação do modelo de precificação de ativos conhecido na literatura de economia financeira como CAPITAL ASSET PRICING MODEL - MODELO DE PRECIFICAÇÃO DE ATIVOS DE CAPITAL, utilizando como ferramentas os balanços patrimoniais das empresas selecionadas. Brasil Ecodiesel indústria e comercio de biocombustíveis e óleos vegetais s.a. (v-agro) e SLC Agrícola s.a. no período relativo ao ano de 2009 e 2010. Resultado fundamental: ambas as empresas no período em estudo apresentaram um beta inferior a 1, ou seja, risco menor que o risco do mercado. Descrevemos alguns resultados estatísticos que mostram o comportamento de ambas as empresas do setor de frigorifico.

Palavras - chaves: commodities agrícolas, CAPM, produtos vegetais.

Abstract: This paper aims to application of CAPITAL ASSET PRICING MODEL using as tools the accounting balances of firms Brasil Ecodiesel indústria e comercio de biocombustíveis e óleos vegetais s.a. (v-agro) e SLC Agrícola s.a in years 2009 and 2010.Fundamental result: both firms in years 22009 - 2010 presented beta value less than 1 , it means risk less than market risk.

Keys - words: agricultural commodities, CAPM, vegetal products.

JEL: A1.

\section{INTRODUÇÃO}

O Brasil tem papel de destaque no segmento de agronegócio relativo a economia mundial. Para se ter uma idéia de tal importância para a economia brasileira, o segmento de agronegócio representa $22 \%$ do produto interno bruto brasileiro.

Agronegócio é um conjunto de atividades que, associadas, garantem a produção, transformação, distribuição e consumo de produtos provenientes da agropecuária. Inclusive, os técnicos da ESALQ/USP, projetam um crescimento no segmento de agronegócio em torno de $40 \%$, nos próximos 10 anos.

Atualmente, o Brasil é o maior exportador de café, açúcar e suco de laranja em escala mundial. Também, tem destaque na exportação de carne bovina, carne suína e frango. Como se percebe, este segmento é um gerador

\footnotetext{
${ }^{1}$ Economista. Doutor em Economia Aplicada pela USP. Professor da Universidade Federal de Sergipe. tacitoaugusto@ufs.br

${ }^{2}$ Economista. Mestrando em Economia pela UFPE. enfigueiredo@yahoo.com.br

${ }^{3}$ Economista. Doutorando em Economia pela ESALQ/USP. fabirosmash@yahoo.com.br
} 
de renda e emprego e, fundamental para o equilíbrio das contas internacionais brasileiras.

Foram escolhidos para propósito de estudo neste artigo os segmentos de biocombustível e oleaginosas, destaques tanto na economia nacional como internacional. Inclusive, nos municípios brasileiros onde prosperam as atividades de agronegócio, o Índice de Desenvolvimento Humano tem revelado um aumento crescente e acentuado na qualidade de vida de seus habitantes.

Os conhecimentos teóricos elaborados e estabelecidos na teoria econômica financeira têm se desenvolvido a passos largos nos últimos 60 anos. Os especialistas se debruçaram no estudo das características e da análise de ativos individuais bem como na constituição e escolha da carteira ótima. Especificamente no artigo em pauta é utilizado as ferramentas conhecidas e fixadas no modelo de equilíbrio no mercado de capitais: modelos de precificação dos ativos financeiros ou modelos de precificação por arbitragem.

Nosso propósito neste artigo é a aplicação direta do modelo de precificação de ativos conhecido na literatura econômica financeira como CAPITAL ASSET PRICING MODEL - "MODELO DE PRECIFICAÇÃO DE ATIVOS DE CAPITAL, desenvolvido por Sharpe(1964), Lintner (1965) e Mossin (1966). Este modelo está inspirado no Princípio da Dominância, em que os ativos que se encontram na FRONTEIRA EFICIENTE têm vantagens sobre os outros ativos no Plano Retornos Esperados e Risco segundo Lencione (2005).

A aplicação está orientada para o setor de commodities agrícolas, segmento fundamental para geração de emprego e renda na economia nacional. $O$ artigo está estruturado da seguinte forma. Seção 2 aponta os elementos orientados para a avaliação de ativos financeiros- valor esperado e desvio padrão; seção 3 apresenta de forma simplificada o CAPM; seção 4, faz uso dos conhecimentos teóricos supra citados no segmento de commodities agrícolas. Ó artigo é finalizado com comentários pertinentes sobre os resultados obtidos.

\section{ELEMENTOS FUNDAMENTAIS PARA AVALIAÇÃO DE ATIVOS FINANCEIROS}

A presente seção se divide da seguinte forma: primeiramente, tratar-se-á do retorno financeiro, como uma variável aleatória; em seguida expor-se-ão diferentes abordagens para o cálculo dos retornos obtidos de ativos financeiros; já a terceira seção utiliza-se dos retornos calculados no passado para demonstrar o conceito de retorno esperado, no qual se baseiam as expectativas dos investidores; na quarta seção, versar-se-á acerca da variância e do desvio-padrão, principais medidas de risco utilizadas; por fim, a quinta e a sexta seção discutem, respectivamente, sobre a definição formal de covariância e a correlação, medidas que determinam o grau de associabilidade entre os títulos financeiros. 


\subsection{O Retorno como Variável Aleatória.}

O retorno financeiro, em termos gerais, pode ser entendido como o montante de ganhos ou perdas vinculados a um ativo dentro de um período específico (GITMAN, 2001). Para o investidor, o retorno está relacionado diretamente ao aumento ou diminuição de sua riqueza, e é certo que os agentes racionais sempre buscam a sua maximização. De todo modo, é necessário tornar esse conceito mensurável, a fim de quantificar a rentabilidade de um determinado ativo.

As variáveis aleatórias são classificadas em discretas e contínuas. Uma v.a é discreta quando o intervalo de valores que ela pode assumir é formado apenas por um número finito de termos, ou infinito enumerável, os quais podem ser contados utilizando-se os números inteiros. Já uma v.a é dita contínua quando ela pode assumir qualquer valor real, dentro de um intervalo (finito ou infinito) de números reais (HSU, 1997).

Considere inicialmente que o retorno de uma determinada ação adquirida por um investidor seja uma v.a discreta denotada por $R$, cujos valores são indicados por $r$ i. Sabemos que não é possível conhecer o retorno exato até sua observação, mas o investidor tem como estimativa suas expectativas dos prováveis retornos. Observando o comportamento da ação ao final de vários meses, o investidor conclui que, no próximo período, o seu retorno somente pode assumir um dentre os seguintes valores: $R_{i}=\{-10 \%$, $5 \%, 15 \%, 30 \%, 40 \%$. Cada um destes valores representa um determinado cenário para o investidor, que pode estar relacionado ao estado em que se encontra a economia, ou mais precisamente, ao desempenho da empresa cuja ação foi adquirida frente a fatores macro ou microeconômicos (SECURATO, 1993). Como, a depender das condições enfrentadas, cada retorno tem uma determinada possibilidade de ocorrer, o investidor formaliza a seguinte idéia quanto ao comportamento da ação:

Tabela 1 - Distribuição probabilística discreta dos retornos

\begin{tabular}{lcc}
\hline Cenário & Retorno $\left(\boldsymbol{R}_{\boldsymbol{i}}\right)$ & Probabilidade $\left(\boldsymbol{P}\left(\boldsymbol{R}_{\boldsymbol{i}}\right)\right)$ \\
\hline Péssimo & $-10 \%\left(r_{1}\right)$ & $10 \%\left(p\left(r_{1}\right)\right)$ \\
Ruim & $-5 \%\left(r_{2}\right)$ & $25 \%\left(p\left(r_{2}\right)\right)$ \\
Regular & $15 \%\left(r_{3}\right)$ & $30 \%\left(p\left(r_{3}\right)\right)$ \\
Bom & $30 \%\left(r_{4}\right)$ & $25 \%\left(p\left(r_{4}\right)\right)$ \\
Excelente & $40 \%\left(r_{5}\right)$ & $10 \%\left(p\left(r_{5}\right)\right)$
\end{tabular}

Fonte: Elaborada pelo autor.

Observe que para cada valor que o retorno possa assumir dentro do intervalo especificado, há uma probabilidade associada. O retorno de $-5 \%$ tem 
$15 \%$ de probabilidade de ocorrer e isso representa um cenário ruim para o investidor (uma recessão econômica ou um desempenho deficiente da empresa em seu setor). Já o retorno de $40 \%$ tem $20 \%$ de probabilidade de se realizar, o que caracteriza um excelente cenário de investimento.

As probabilidades do retorno deste exemplo são definidas como probabilidades objetivas ${ }^{4}$ : é a freqüência relativa com que um determinado retorno (evento) ocorre em muitas observações deste experimento não controlado (o comportamento da ação). Assim, temos que, se $t$ é a quantidade de vezes em que o retorno da ação foi observado ao final de um mês, e $t\left(R_{i}\right)$ é o número de vezes que um determinado retorno ocorre durante essas observações, a probabilidade de $R_{i}$ é dada pelo quociente $\frac{t\left(R_{i}\right)}{t}$ quando $t$ tende ao infinito:

$$
P\left(R_{i}\right)=\lim _{t \rightarrow \infty} \frac{t\left(R_{i}\right)}{t}
$$

O conjunto de valores discretos que o retorno pode assumir, juntamente com suas respectivas probabilidades de ocorrência, como mostra a tabela 1 , formam a chamada distribuição probabilística discreta dos retornos, à qual está associada uma função distribuição de probabilidade ou função massa de probabilidade dos retornos (HOGG et al., 2005). Para a v. a discreta $R_{i}$, o valor da sua função massa de probabilidade, dada por $p\left(r_{i}\right)$, é a probabilidade de $R_{i}$ tomar um determinado valor $r_{i}$ :

$$
p\left(r_{i}\right)=P\left(R_{i}=r_{i}\right)
$$

Considerando os retornos discretos supra-elencados, o valor da função distribuição de probabilidade de $r_{2}$, por exemplo, é tal que $p(-5 \%)=P\left(R_{i}=-5 \%\right)=20 \%$.

A função massa de probabilidade de uma v.a discreta deve atender certas premissas básicas (ROSS, 2003). Primeiramente, o valor de $p\left(r_{i}\right)$ é sempre positivo para todos os valores discretos de $R_{i}$ e se encontra no intervalo $[0,1]$. Isto é, se $R_{i}$ assume um dos valores $r_{1}, r_{2}, \ldots, r_{n}$, então:

\footnotetext{
${ }^{4}$ Segundo ASSAF NETO (2005, p. 320), "a probabilidade objetiva pode ser definida a partir de séries históricas de dados e informações, freqüências relativas observadas e experiência acumulada no passado". Por outro lado, poderíamos considerar também a probabilidade subjetiva do investidor, que "tem como base a intuição, o conhecimento, a experiência do investimento e, até mesmo, um certo grau de crença na unidade tomadora de decisão" (idem).
} 


$$
\begin{array}{r}
0 \leq p\left(r_{i}\right) \leq 1, i=1,2, . ., n \\
p\left(r_{i}\right)=0, i \neq 1,2, . ., n
\end{array}
$$

Além disso, a função massa de probabilidade distribui o total de 1 unidade de probabilidade por todo o conjunto de valores que a v.a discreta pode assumir. Significa que a soma dos valores de $p\left(r_{i}\right)$ para todo o conjunto de valores de $R_{i}$ deve ser igual a 1 :

$$
p\left(r_{1}\right)+p\left(r_{2}\right)+\ldots p\left(r_{n}\right)=\sum_{i=1}^{t} p\left(r_{i}\right)=1
$$

A distribuição probabilística dos retornos da ação do nosso exemplo pode ser ilustrada no gráfico a seguir. No eixo horizontal temos os valores de $R_{i}$, dados por $r_{1}$ a $r_{5}$, enquanto que no eixo vertical temos os valores da função massa de probabilidade para cada ocorrência.

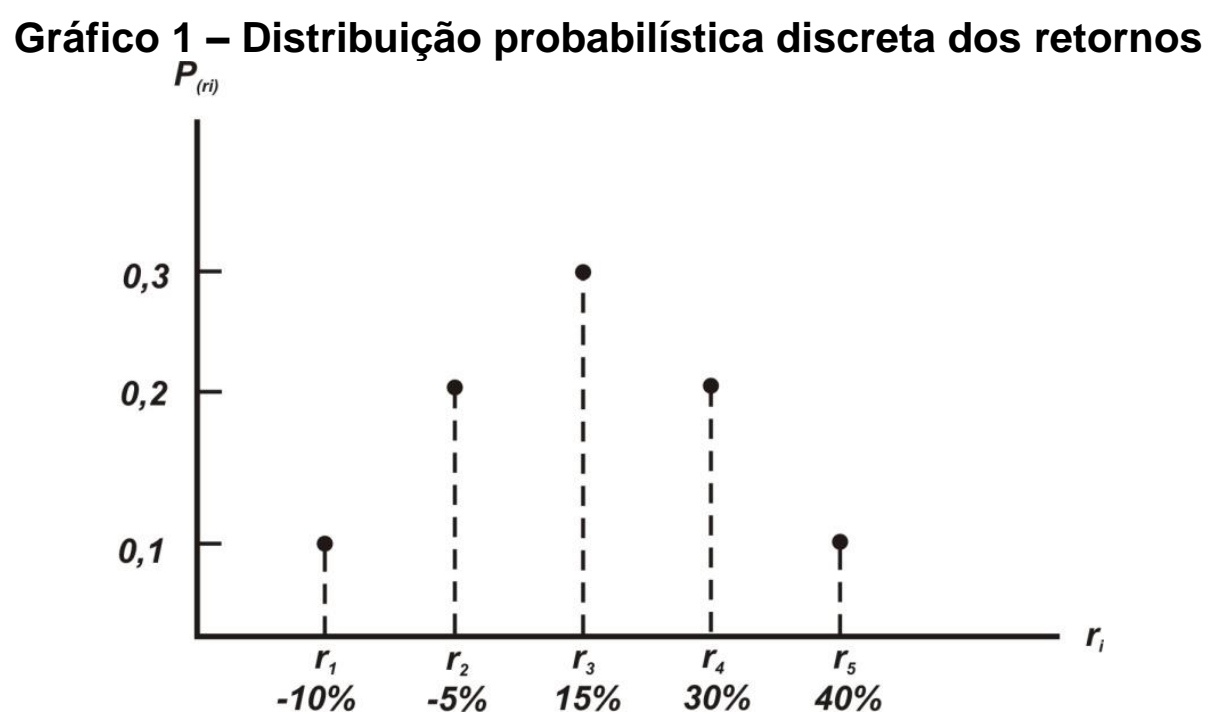

Fonte: Elaborado pelo autor.

Ao se trabalhar empiricamente com a série de retornos de um ativo financeiro, é necessário, portanto, definir-se previamente o espectro probabilístico que guiará a análise: se os retornos serão avaliados como discretos ou contínuos. Geralmente, trabalha-se com retornos na sua forma discreta, na medida em que esta abordagem permite uma maior simplificação dos cálculos. Entretanto, a abordagem contínua é mais realista, principalmente se estamos lidando com uma grande amostra de observações, porquanto os retornos contínuos permitem a construção de uma distribuição de probabilidade economicamente mais significante (JORION, 1998). 


\subsection{Os Retornos Obtidos.}

Para efeito de classificação das diferentes espécies de retorno financeiro, costuma-se dividir os ativos negociados no mercado de capitais em instrumentos de renda fixa e de renda variável. Os títulos de renda fixa oferecem uma taxa prometida de retorno, associada a uma determinada taxa de juros - existe, portanto, um parâmetro que define sua rentabilidade. Já os títulos de renda variável, tais como as ações, não apresentam uma taxa prometida de retorno, ou seja, não há a promessa de pagamento de dinheiro no futuro (BODIE e MERTON, 2002).

De fato, quando o investidor aplica o seu capital em títulos acionários, o retorno obtido pela aplicação do capital pode ser decomposto em dois componentes. O primeiro consiste no pagamento de dividendos aos acionistas. Os dividendos dependerão dos critérios definidos pela empresa, a depender do lucro arrematado, e por isso não há garantia de recebimento. Portanto, como não existe uma taxa prometida de retorno, os dividendos não podem ser considerados como juros. Já o segundo componente diz respeito aos ganhos ou perdas no preço de mercado das ações dentro de um determinado período (dias, meses, anos, etc), sendo esse retorno chamado de ganho ou perda de capital $^{5}$ (ROSS et. al, 2002).

Assim, definindo-se um determinado horizonte de mensuração, como um mês, por exemplo, os retornos absolutos obtidos pela realização de investimentos em ativos financeiros são dados pela diferença entre o valor do título ao final do mês corrente, denotado por $P_{t}$, e o valor do título ao final do mês anterior, denotado por $P_{t-1}$. Já o retorno percentual, ou taxa de retorno discreta, de um determinado título $i$, é definido como o ganho de capital ocorrido durante esse período, considerando-se discretos os valores da variável aleatória $R_{i}$ (COSTA e ASSUNÇÃO, 2005):

$$
R_{i}=\frac{P_{t}-P_{t-1}}{P_{t}}
$$

Para investimentos em ações, as quais podem oferecer dividendos, denotados por $D_{i}$, e desconsiderando os efeitos inflacionários, tem-se que a taxa de retorno é dada por (BODIE e MERTON, 2002):

$$
R_{i}=\frac{P_{t}-P_{t-1}+D_{t}}{P_{t}}
$$

\footnotetext{
${ }^{5}$ As perdas de capital são também chamadas de ganhos negativos de capital.
} 
É possível também representar a taxa de retorno através de seus dois componentes, o dividendo e a variação no preço de mercado da ação:

$$
R_{i}=\frac{D_{t}}{P_{t}}+\frac{P_{t}-P_{t-1}}{P_{t}}
$$

As definições (2.10) e (2.11) implicam, necessariamente, que qualquer rendimento advindo de dividendos será reinvestido no final do mês, na compra de mais ações.

Se, por outro lado, estivermos tratando com retornos de horizonte longo, é mais conveniente considerar os valores obtidos de $R_{i}$ como contínuos. Assim, a taxa de retorno continuamente composta de $R_{i}$, também chamada de logretorno, é definida em termos do logaritmo da razão do preço (MORETTIN e TOLOI, 2006):

$$
R_{i}=\ln \left(\frac{P_{t}}{P_{t-1}}\right)=\ln P_{t}-\ln P_{t-1}
$$

Com o adendo de dividendos, a taxa de retorno contínua é dada por:

$$
R_{i}=\ln \left[\frac{\left(P_{t}+D_{t}\right)}{P_{t-1}}\right]=\ln \left(P_{t}+D_{t}\right)-\ln P_{t-1}
$$

Uma das vantagens de se utilizar retornos contínuos é o fato de permitirem, com facilidade, extensões para múltiplos períodos. Considere, por exemplo, o retorno sobre um período de três meses. O retorno logaritmo pode ser decomposto como:

$$
R_{i, 3}=\ln \left(\frac{P_{t}}{P_{t-3}}\right)=\ln \left(\frac{P_{t}}{P_{t-1}}\right)+\ln \left(\frac{P_{t-1}}{P_{t-2}}\right)+\ln \left(\frac{P_{t-2}}{P_{t-3}}\right)=R_{t-2}+R_{t-1}+R_{t}
$$

O que é particularmente conveniente, já que o retorno contínuo de três meses é simplesmente a soma de três retornos mensais.

Por fim, pode o investidor desejar conhecer o seu retorno acumulado, aquele que ganharia caso mantivesse seu investimento por vários períodos seguidos. Considerando o rendimento obtido a cada mês de aplicação, o retorno acumulado ao final de $t$ meses é tal que: 


$$
R_{a c m}=\left[\prod_{i=1}^{t}\left(1+R_{i}\right)\right]-1
$$

A depender do tratamento despendido aos dados, há, como elencado, diversas formas de se calcular o retorno realizado de um título financeiro, seja ele de risco ou não. O cálculo dos retornos obtidos em períodos passados, como veremos, é de grande importância para a construção das expectativas quanto ao futuro desempenho do investimento empreendido.

\subsection{O Retorno Esperado de Ativos Financeiros}

Através do conjunto de preços e dividendos passados de um ativo financeiro, é possível construir a série histórica de retornos realizados, fixandose para tanto uma janela temporal de análise. Entretanto, para o investidor, o importante é a sua rentabilidade futura, aquela que será obtida após o momento em que se decide investir. Apesar de não ser possível prever o retorno futuro com exatidão, é necessário que o investidor detenha uma estimativa dos próximos retornos, a fim de auxiliá-lo na tomada de decisão quanto à alocação de seu capital. Nesse sentido, a informação acerca do comportamento anterior de um ativo, representado na forma do cálculo dos retornos ocorridos no passado, serve de base para o investidor formar suas expectativas quanto ao futuro desempenho do seu investimento.

Em linhas gerais, o retorno que o investidor espera obter no futuro, ou no período seguinte ao seu investimento, é definido como retorno esperado (JORION, 1998). Na análise, o retorno esperado de um ativo financeiro é obtido através da utilização de uma das principais características de uma variável aleatória: a esperança matemática, também chamada de valor esperado, primeiro momento, ou valor médio da variável aleatória. A esperança matemática é o valor de tendência central da distribuição de probabilidade de uma variável aleatória, ou seja, é o valor médio que uma v.a assume em um número infinito de repetições de um experimento. Se observarmos um experimento um grande número de vezes, a v.a tenderá a assumir determinado valor, sendo este o seu valor esperado ou valor médio.

Considere que o retorno $R_{i}$ de uma ação é uma v.a discreta que pode tomar os valores $r_{1}, r_{2}, \ldots, r_{n}$, com probabilidades $p\left(r_{1}\right), p\left(r_{2}\right), p\left(r_{n}\right)$, obtidas pela sua função massa de probabilidade $p\left(r_{i}\right)$. A sua esperança matemática, ou valor esperado, denotado por $E\left(R_{i}\right)$, é dado por (ROSS, 2003):

$$
E\left(R_{i}\right)=r_{1} p\left(r_{1}\right)+r_{2} p\left(r_{2}\right)+\ldots+r_{n} p\left(r_{n}\right)=\sum_{i=1}^{t} r_{i} p\left(r_{i}\right)
$$


Onde $E\left(R_{i}\right)$, como retorno médio, é frequentemente indicado por $\mu$ :

$$
E\left(R_{i}\right)=\mu
$$

A equação acima na mostra que o retorno esperado é uma média ponderada dos valores discretos que $R_{i}$ pode assumir, tendo como ponderações as probabilidades associadas a cada ocorrência. Dada uma série de retornos efetivos passados, variando do período 1 a $t$, o retorno que 0 investidor espera obter no próximo período, $t+1$, é igual ao somatório do produto desses retornos pelas suas respectivas probabilidades.

A esperança matemática possui algumas propriedades úteis, que valem tanto se o retorno de um ativo for considerado uma v.a discreta ou contínua. Temos que, se $c$ é uma constante que multiplica $R_{i}$, o valor esperado é igual a $c$ vezes o retorno esperado:

$$
E\left(c R_{i}\right)=c E\left(R_{i}\right)
$$

Ademais, o valor esperado da soma ou da diferença de dois retornos $R_{i}$ e $R_{j}$ é igual à soma ou a diferença entre os próprios retornos esperados:

$$
E\left(R_{i} \pm R_{j}\right)=E\left(R_{i}\right) \pm E\left(R_{j}\right)
$$

Em todo caso, o retorno esperado de um ativo costuma ser estimado, na prática, pressupondo-se que em toda a população de observações passadas os retornos apresentam a mesma probabilidade de ocorrência. Ou seja, pressupõe-se que os retornos são identicamente distribuídos, equiprováveis. Este método é aplicado visto que, geralmente, não se tem acesso à verdadeira distribuição probabilística dos retornos. Nesse caso, se $t$ é a quantidade de retornos observados, o retorno esperado, ou primeiro momento $\mu=E\left(R_{i}\right)$, pode ser estimado pela média amostral dos retornos passados:

$$
\hat{\mu}=\bar{R}_{i}=\frac{\sum_{i=1}^{t} r_{i}}{t}
$$

Onde se assume que $\hat{\mu}$ se aproxima de $\mu$, o verdadeiro valor esperado da variável aleatória $R_{i}$. Em suma, estamos considerando que o investidor tem como estimativa do retorno futuro de sua ação a média dos retornos que o título tenha obtido no passado. 
Entretanto, devemos ter em mente que, como se trata de uma expectativa, o retorno que efetivamente ocorrerá poderá ser superior ou inferior ao retorno esperado. Cabe ao investidor, portanto, determinar o método no qual suas expectativas estarão baseadas, a fim de captar ao máximo o movimento futuro do ativo. Geralmente, assume-se que o retorno esperado de um título seja igual ao retorno médio que este tenha apresentado passado, como foi aqui desenvolvido. Não obstante, outros métodos podem ser considerados, como a aplicação de modelos mais robustos de previsão ou mesmo se o investidor possui determinada informação privilegiada que possa auxiliá-lo na formação de suas expectativas (ROSS et al., 2002).

\subsection{Medidas de Risco}

O conceito de risco financeiro atinge as mais variadas esferas da sociedade, sejam empresas, indivíduos ou governo. De fato, toda empresa tem seu risco financeiro e os seus negócios estão intrinsecamente ligados à administração do risco (JORION, 1998). Da mesma forma, os investidores individuais incorrem em risco quando da formulação de suas carteiras, independente da soma de capital que despendam na sua composição. A busca de rendimentos oriundos de ativos financeiros traz, inevitavelmente, um determinado risco associado. Assim, os agentes que estejam atuando no mercado de capitais, supondo-os racionais e avessos à volatilidade de seus investimentos, devem buscar maximizar o seu retorno dentro de um nível de risco aceitável.

$\mathrm{Na}$ literatura, apesar do risco ser tratado sob diferentes abordagens, todas convergem para o entendimento de que o mesmo é algo desfavorável, prejudicial e contrário ao interesse esperado pelo agente. JORION (1998, p.3) define o risco como a "volatilidade de resultados inesperados, normalmente relacionada ao valor de ativos ou passivos de interesse". Já MERTON E BODIE (2002, p. 258) tratam o risco sob o ponto de vista da incerteza; para os autores a incerteza "existe sempre que não se sabe ao certo o que vai ocorrer no futuro". Sendo assim, "o risco é a incerteza que importa, porque afeta o bem-estar das pessoas", já que "toda situação de risco é incerta, mas pode haver incerteza sem risco" (idem). ROSS et al. (2002, p. 194-195) reitera que não há uma definição de risco universalmente aceita, mas propõe que uma das maneiras de se pensar o risco em ativos financeiros é analisar o grau de dispersão da distribuição de frequência dos retornos: "a dispersão de uma distribuição é uma medida de quanto um dado retorno pode se afastar do retorno médio. Se a distribuição apresentar uma dispersão muito grande, os retornos que poderão ocorrer serão muito incertos". Em outras palavras, podese inferir que a incerteza e o risco estão atrelados e, portanto, retornos incertos podem ser entendidos como retornos arriscados.

O desvio-padrão e a variância são as medidas de dispersão utilizadas para se auferir estatisticamente o risco. Supondo um determinado ativo financeiro com retorno $R_{i}$, a sua variância, denotada por $\operatorname{Var}\left(R_{i}\right)$ ou $\sigma^{2}$, é dada 
pelo segundo momento da distribuição de probabilidade das observações. Ou seja, é a diferença quadrática média entre os valores assumidos por $R_{i}$ e o seu valor esperado:

$$
\operatorname{Var}\left(R_{i}\right)=\sigma^{2}=E\left[R_{i}-E\left(R_{i}\right)\right]^{2}=E\left[R_{i}^{2}\right]-\left[E\left(R_{i}\right)\right]^{2}
$$

Assim, temos que a variância do retorno é a média ponderada dos quadrados das diferenças (ou distâncias) entre os valores que $R_{i}$ pode tomar e o seu valor esperado, ou médio (o centro da função de distribuição de probabilidade). Quanto maior a variância de um título, maior será a distância quadrática média entre os valores do retorno e sua média, ou seja, mais dispersos serão os rendimentos - o que representa um maior risco incorrido.

Uma propriedade muito útil das variâncias é que, se $c$ é uma constante que multiplica o retorno, a variância será igual ao quadrado da constante vezes a variância do retorno:

$$
\operatorname{Var}\left(c R_{i}\right)=c^{2} \operatorname{Var}\left(R_{i}\right)
$$

Para o cálculo efetivo da variância, devemos antes estabelecer se 0 retorno do ativo se comporta de forma contínua ou discreta. Considerando o retorno como uma v.a discreta com valores $r_{i}$, com função massa de probabilidade $p\left(r_{i}\right)$, a variância é dada por:

$$
\sigma^{2}=\sum_{i=1}^{t}\left[r_{i}-E\left(R_{i}\right)\right]^{2} p(r i)
$$

Se o cálculo da variância for realizado com base na população de retornos passados, onde se admite que todos os valores têm a mesma probabilidade de ocorrência, a variância populacional da distribuição de retornos pode ser estimada da seguinte forma:

$$
\sigma^{2}=\frac{\sum_{i=1}^{t}\left(r_{i}-\bar{R}_{i}\right)^{2}}{t}
$$

Quando o cálculo for realizado considerando-se apenas uma amostra da população, um estimador não tendencioso da variância amostral poderá ser calculado do seguinte modo: 


$$
\sigma^{2}=\frac{\sum_{i=1}^{t}\left(r_{i}-\bar{R}_{i}\right)^{2}}{t-1}
$$

A equação (2.28) pode ser expandida da seguinte forma:

$$
\sigma^{2}=\frac{1}{t-1} \sum_{i=1}^{t} r_{i}^{2}-\frac{t}{t-1} \bar{R}_{i}^{2}
$$

O que nos mostra que a variância é composta por dois termos: o primeiro é a média dos retornos quadrados e o segundo é o quadrado da média.

No entanto, o fato de a variância ser medida em quadrados torna esta medida um número grande e de difícil manejo, pois seu valor sai dos limites dos valores observados em um conjunto de dados. Além disso, os retornos obtidos ao se investir em um título não são calculados em termos quadráticos, como se demonstra nas equações (2.10) a (2.16). Necessita-se, pois, de uma medida representativa de risco que esteja na mesma escala dos retornos financeiros. Isto pode ser solucionado através da utilização do conceito de desvio-padrão. O desvio-padrão é nada mais do que a raiz-quadrada da variância, geralmente representado por $\mathrm{DP}\left(R_{i}\right)$ ou $\sigma$ :

$$
D P\left(R_{i}\right)=\sigma=\sqrt{\frac{\sum_{i=1}^{t}\left(r_{i}-\bar{R}_{i}\right)^{2}}{t-1}}
$$

Ao se retirar a raiz quadrada da variância, o valor resultante adentra novamente nos limites dos retornos observados da amostra. Nesse sentido, o desvio-padrão representa o erro médio obtido ao se substituir cada valor do conjunto de dados pela média amostral (no caso dos retornos, o erro médio gerado ao se substituir cada valor $r_{i}$ pelo retorno médio $\bar{R}_{i}$ ).

Uma propriedade do desvio-padrão que deriva da variância é que, considerando uma constante $c$ que multiplica o retorno, o desvio-padrão é igual à constante vezes o desvio-padrão do retorno:

$$
D P\left(c R_{i}\right)=c D P\left(R_{i}\right) \mathrm{n}
$$


Isto resulta da equação (2.24) e do fato de que a raiz quadrada do produto de dois números é o produto de cada um dos números.

Observa-se, portanto, que pelo fato do desvio-padrão ser expresso na mesma unidade escalar da variável analisada, esta medida pode ser utilizada para melhor descrever a quantidade de dispersão na distribuição dos retornos, representando assim o risco da ação 6 .

\subsection{Covariância e Correlação}

A covariância é uma medida que expressa a intensidade com que duas variáveis aleatórias estão associadas - no caso aqui proposto, o grau de interdependência entre as taxas de retorno de duas ações. Nesse sentido, a covariância é uma medida de análise que expressa a expectativa do investidor quanto ao movimento conjunto de pares de títulos.

Considerando dois ativos $A$ e $B$ com retornos respectivamente expressos por $R_{A}$ e $R_{B}$, a covariância entre ambos, denotada por $\sigma_{A, B}$ ou COV( $R_{A}, R_{B}$ ), é dada por (HOGG et al., 2005):

$$
\begin{aligned}
\operatorname{COV} & \left(R_{A}, R_{B}\right)=\sigma_{A, B}=E\left[\left(R_{A}-E\left(R_{A}\right)\right)\left(R_{A}-E\left(R_{B}\right)\right)\right] \\
& =E\left(R_{A} R_{B}-E\left(R_{B}\right) R_{A}-E\left(R_{A}\right) R_{B}+E\left(R_{A}\right) E\left(R_{B}\right)\right) \\
& =E\left(R_{A} R_{B}\right)-E\left(R_{B}\right) E\left(R_{A}\right)-E\left(R_{A}\right) E\left(R_{B}\right)+E\left(R_{A}\right) E\left(R_{B}\right) \\
& =E\left(R_{A} R_{B}\right)-E\left(R_{A}\right) E\left(R_{B}\right)
\end{aligned}
$$

Onde a ordem das variáveis na fórmula da covariância não interfere no resultado. Em outras palavras, a covariância de $A$ com $B$ é a mesma de $B$ com A: $\operatorname{Cov}\left(R_{A}, R_{B}\right)=\operatorname{Cov}\left(R_{B}, R_{A}\right)$ ou $\sigma_{A, B}=\sigma_{B, A}$. A covariância, portanto, representa a produto das diferenças entre os valores do retorno de cada ação pelos seus respectivos valores esperados. 7

Ross et al. (2002) explica melhor a intuição por trás da definição da covariância. Suponha que a taxa de retorno do título $A$ seja geralmente superior à sua média quando a taxa de retorno do título $B$ também seja superior à sua média, ou que o retorno do título $A$ esteja frequentemente abaixo de sua média quando o retorno do título $B$ também esteja abaixo de sua média. Essa situação indicaria uma relação de dependência positiva entre os ${ }^{6}$ O desvio-padrão é costumeiramente chamado de volatilidade, principalmente em se tratando de retornos
contínuos (JORION, 1998). 
retornos das duas ações, visto que a expressão que representa a covariância torna-se sempre positiva quando ambos os retornos estiveram abaixo ou acima de suas médias.

Por outro lado, se a taxa de retorno do título $A$ estiver geralmente abaixo de sua média quando a taxa de retorno do título $B$ estiver acima de sua média, ou vice-versa, haverá uma relação de dependência negativa entre os retornos destas duas ações. Isso se reflete na expressão da covariância, que será sempre negativa quando um retorno estiver acima de sua média enquanto 0 outro estiver abaixo de sua média.

Por último, tem-se a situação em que as taxas de retorno dos títulos não estão relacionadas. $O$ fato do retorno do título $A$ estar acima ou abaixo de sua média nada nos informa a respeito do movimento do retorno do título $B$. Como não haverá tendência de os retornos serem positivos ou negativos, estes tenderão, em média, a se contrabalancear e se anular, tornando a covariância igual à zero.

Com relação a este último caso, ROSS et al (2002) explica que, ao analisar uma série histórica financeira, é improvável que a fórmula da covariância apresente resultado exatamente igual a zero. Para o autor, isso se deve a erro de amostragem, pois o mero caráter aleatório da série faz com que o resultado acabe sendo positivo ou negativo. De todo modo, o valor esperado da covariância para uma amostra histórica suficiente grande, quando as taxas de retorno dos títulos não estão associadas, é, de fato, bem próximo de zero.

Entretanto, do mesmo modo que a variância, a covariância é medida em quadrados de diferenças, o que torna o seu valor numérico de difícil interpretação. Tal problema é resolvido com o cálculo do coeficiente de correlação, que nada mais é do que a covariância entre o retorno de dois títulos dividido pelo produto dos seus respectivos desvios-padrão, como veremos a seguir.

A correlação, ou coeficiente de correlação, é uma medida estatística que indica o grau de dependência linear entre duas variáveis aleatórias (HOGG et al., 2005). A correlação apresenta as mesmas propriedades da covariância, com a vantagem de ser uma medida adimensional e normalizada, o que permite uma melhor interpretação da relação de associabilidade entre duas variáveis. Por este motivo, a correlação é muitas vezes utilizada em detrimento da covariância para se analisar o risco de um portfólio, como será visto na próxima seção. Além disso, o coeficiente de correlação é importante para o delineamento da fronteira eficiente de Markowitz, bem como para o processo de otimização de portfólios.

Formalmente, o coeficiente de correlação entre duas variáveis aleatórias, tais quais os retornos de duas ações $A$ e $B$, frequentemente indicado por $\rho_{A, B}$ ou $\operatorname{CORR}\left(R_{A}, R_{B}\right)$ é definido como ${ }^{7}$ :

\footnotetext{
${ }^{7} \mathrm{Na}$ realidade, existem diferentes tipos de coeficientes de correlação, cada qual utilizado para uma situação de análise específica. O mais conhecido é coeficiente de correlação de Pearson, também chamado de coeficiente de correlação produto-momento ou "r de Pearson", que é obtido justamente ao se dividir a covariância de duas variáveis pelos seus respectivos desvios-padrão. Apesar do seu nome, quem utilizou pela primeira vez o coeficiente de Pearson foi o estatístico Francis Galton em 1888
} 


$$
\begin{gathered}
\operatorname{CORR}\left(R_{A}, R_{B}\right)=\rho_{A, B}=\frac{E\left[\left(R_{A}-E\left(R_{A}\right)\right)\left(R_{A}-E\left(R_{B}\right)\right)\right]}{\sqrt{E\left[R_{A}-E\left(R_{A}\right)\right]^{2}} \sqrt{E\left[R_{B}-E\left(R_{B}\right)\right]^{2}}} \\
=\frac{E\left(R_{A} R_{B}\right)-E\left(R_{A}\right) E\left(R_{B}\right)}{\sqrt{E\left[R_{A}^{2}\right]-\left[E\left(R_{A}\right)\right]^{2}} \sqrt{E\left[R_{B}^{2}\right]-\left[E\left(R_{B}\right)\right]^{2}}}
\end{gathered}
$$

E, a partir das definições (2.23) e (2.32), tem-se que:

$$
\rho_{X, Y}=\frac{\operatorname{Cov}(X, Y)}{\sigma_{X} \sigma_{Y}}
$$
sorte que:

Pode-se também expressar a covariância em termos da correlação, de

$$
\operatorname{Cov}(X, Y)=\rho_{X, Y} \sigma_{X} \sigma_{Y}
$$

Tal como na covariância, a ordem das duas variáveis é irrelevante para o cálculo da correlação, ou seja: $\operatorname{Corr}(X, Y)=\operatorname{Corr}(Y, X)$ ou $\rho_{X, Y}=\rho_{X, Y}$. Além disso, visto que o desvio-padrão de uma variável é sempre positivo, o sinal da correlação é o mesmo do da covariância.

A divisão da covariância pelo produto dos desvios-padrão constitui-se em um processo de padronização, o que faz com que o coeficiente de correlação resulte sempre em um valor entre -1 e +1 . Dessa forma, 0 coeficiente de correlação indica não somente a força com que duas variáveis estão relacionadas, mas também a direção dessa associação.

Assim, uma correlação igual a +1 significa uma associação positiva perfeita entre duas variáveis, ou seja, há uma relação linear crescente proporcional entre ambas. Já uma correlação igual a -1 caracteriza uma associação negativa perfeita entre duas variáveis, isto é, uma relação linear proporcionalmente inversa entre elas. Se a correlação for igual a zero significa que as duas variáveis não dependem linearmente uma da outra, são não correlacionadas; no entanto, deve-se examinar esta situação com maior acuidade, pois quando o valor do coeficiente de correlação for igual a zero 
pode haver outra espécie de associação, como uma dependência não-linear, entre as duas variáveis (MAKRIDAKIS et al., 1983). Qualquer outro valor entre -1 e +1 indica que as variáveis são positivamente ou negativamente correlacionadas, a depender do sinal, sendo tão mais forte o grau de associação quanto mais próximo estiver dos valores extremos.

Conforme explica SAMANEZ (2007), é muito difícil encontrar correlações perfeitamente positivas, negativas ou nulas entre o retorno de pares de ações, visto que geralmente são positivas ou ligeiramente negativas. BRIGHAM e HOUSTON (1999), por sua vez, informam que ações apresentam coeficiente de correlação por volta de 0,6 na maioria dos casos, sendo que inexistem ativos no mercado com correlação negativa perfeita. De todo modo, o entendimento dos valores assumidos pelo coeficiente de correlação é muito importante para o processo de diversificação de carteiras, dada a possibilidade de comparar a correlação de pares distintos de títulos, a fim de julgar aqueles que melhor contribuem para a redução do risco do portfólio.

Os gráficos 3, 4 e 5 abaixo mostram a evolução dos retornos dos ativos $A$ e $B$ no tempo, para situações em que a correlação for, respectivamente, positiva perfeita, negativa perfeita e nula. Observe que no gráfico 3 os retornos de $A$ e $B$ são, ao mesmo tempo, superiores ou inferiores à média, enquanto que no gráfico 4 o título $A$ apresenta retorno superior a média quando o título $B$ possui retorno inferior à média e vice-versa. Já no gráfico 5 o retorno do título $A$ não é relacionado linearmente com retorno do título $B$, pois o fato do retorno de $A$ estar acima da média em nada nos informa sobre o movimento do retorno de $B$.

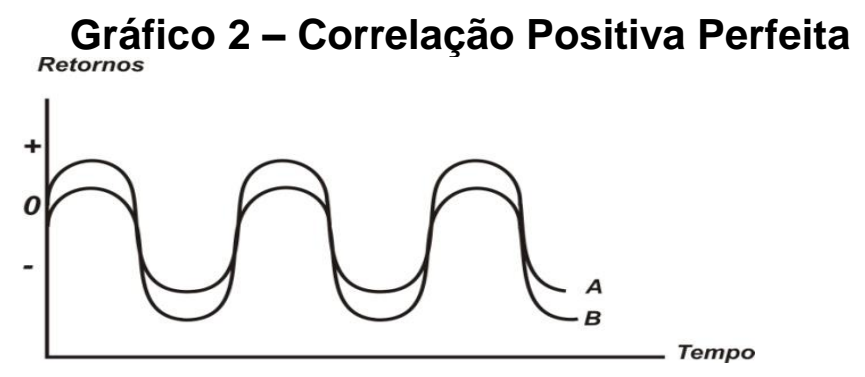

Fonte: Elaborada pelo autor.

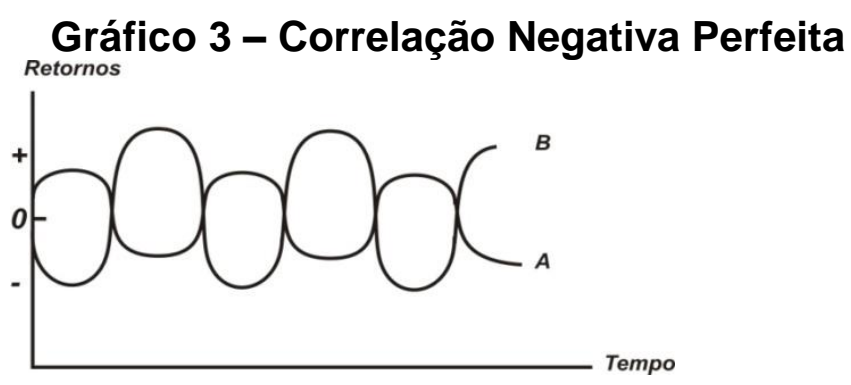

Fonte: Elaborada pelo autor. 


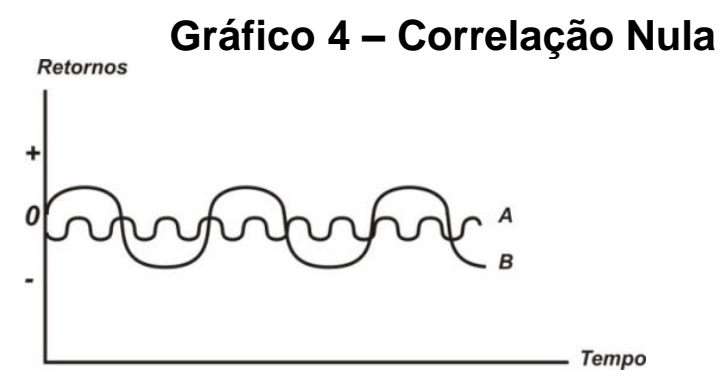

Fonte: Elaborada pelo autor.

Após apresentar nesta seção, os elementos de retorno e risco de títulos individuais, além da relação de dependência entre o retorno de ativos, cabe agora destacar como estes conceitos se incorporam no modelo de CAPM.

\section{MODELO DE PRECIFICAÇÃO DE ATIVOS FINANCEIROS - CAPM.}

Mensurar o risco não é tarefa simples. A matemática para o cálculo do risco exige um conhecimento da teoria da probabilidade e de como os riscos e retornos dos ativos se comportam dentro de um modelo significativo. Um dos mais bem sucedidos modelos de mensuração do risco é denominado CAPM (GROPPELLI, 1998).

O modelo de precificação de ativos financeiros (CAPM) relaciona os riscos sistemáticos, ou não diversificáveis, aos retornos esperados de um projeto. Apesar de ser mais indicado para a análise de ativos financeiros, também pode ser utilizado na avaliação de risco e retorno de investimentos e ativos empresariais (GROPPELLI, 1998). Como também na determinação do custo de capital próprio. (MARTERLANC, 2005)

O custo de capital é a taxa mínima de retorno necessária para atrair investidores. Em outras palavras, é a taxa que o investidor pode obter em outras modalidades semelhantes de investimento. Outra maneira de destacar o custo de capital é como sendo a taxa de retorno que torna indiferente, para o investidor a aprovação ou não de um projeto. (MARTERLANC, 2005)

De acordo com as definições de custo de capital, podem-se observar as inúmeras aplicabilidades da análise do custo de capital: Avaliação do desempenho empresarial; avaliação de investimentos; avaliação de decisões de financiamento; nas operações da empresa; e principalmente na escolha de uma carteira de ativos (MARTERLANC, 2005)

O pressuposto básico do CAPM é a existência de um relacionamento próximo entre os retornos dos ativos individuais e os retornos do mercado. Os retornos de uma carteira de ativos consistem nos ganhos de capital e das receitas com dividendos. Também é destacado que o mercado de ativos é 
altamente eficiente e de assimilação rápida de todas as informações disponíveis. (GROPPELLI, 1998)

Assim, devido a esse relacionamento próximo, a instabilidade do mercado nos apresenta um coeficiente comum para a determinação do grau de risco de um ativo individual. Esse grau de risco é definido pelo nível de sensibilidade dos retornos de um ativo em relação ao mercado. Dessa forma, relaciona um índice comum medido pela sensibilidade do ativo individual em confronto com um índice comum, caracterizado pelo mercado. Quando o retorno do ativo sobe ou desce numa proporção maior que o do mercado, indica que o ativo possui risco maior que o mercado. Se o retorno do ativo sobe ou desce numa proporção menor que o do mercado, diz-se que o ativo possui risco menor que o do mercado. Pode-se, também, classificar os riscos de vários ativos apenas relacionando-os ao índice comum do mercado. (GROPPELLI, 1998)

Mas como determinar a sensibilidade de um ativo em relação ao mercado?

A sensibilidade de um ativo em relação ao mercado pode ser determinada, de forma simplificada, pela comparação entre os desvios-padrão do ativo e do mercado, tal que: (GROPELLI, 1998)

$$
\text { Sensibilidade }=\frac{\text { volatilidade dos retornos do ativo } j\left(\sigma_{j}\right)}{\text { volatilidade dos retornos do mercado } m\left(\sigma_{m}\right)}
$$

O modelo CAPM aborda, de forma mais completa, a sensibilidade através da análise do coeficiente beta $(\beta)$, porém o conceito é bastante semelhante. (GROPPELLI, 1998).

O coeficiente beta $(\beta)$ pode ser considerado o coeficiente do risco específico de um ativo em relação ao índice representativo do mercado. No caso de uma firma com expressiva participação na bolsa de valores, o beta de sua ação pode ser determinado pela regressão de seus retornos periódicos em relação ao índice de mercado selecionado em períodos anteriores. (MARTELANG, 2005)

No modelo CAPM, o coeficiente beta $(\beta)$ é dado pela variação entre os retornos dos ativos, caracterizados por $\left(R_{j}\right)$ e os retornos médios do mercado $\left(R_{m}\right)$. Esse valor de beta $(\beta)$ mede necessariamente a sensibilidade ou risco de um ativo em relação ao mercado. Estatisticamente pode-se escrever essa relação na forma de uma linha característica, como descrita abaixo. (GROPPELLI, 1998).

$$
R_{j}=a+\beta R_{m}+e
$$


Onde, $\beta$ é o coeficiente de sensibilidade, $R_{m}$ é o retorno médio do mercado, $R_{j}$ é o retorno do ativo j, $a$ é um constante e $e$ é o erro.

Outra maneira de definirmos o valor de beta $(\beta)$ é como a medida de volatilidade dos retornos de um título com relação aos retornos do mercado como um todo, podendo ser calculado pela fórmula: (Martelanc, 2005)

$$
\beta_{j}=\frac{\operatorname{Cov}\left(R_{j}, R_{m}\right)}{\sigma^{2}\left(R_{m}\right)} \text { ou } \rho\left(R_{j}, R_{m}\right) \cdot \frac{\sigma R_{j}}{\sigma R_{m}}
$$

Onde, $\beta_{j}$ é o beta do ativo j, $\operatorname{Cov}\left(R_{j}, R_{m}\right)$ é a covariância entre os retornos do ativo j e a carteira do mercado, $\sigma^{2}\left(R_{m}\right)$ é a variância dos retornos da carteira de mercado, $\rho\left(R_{j}, R_{m}\right)$ é o coeficiente de correlação entre os retornos dos ativos j da carteira de mercado $\mathrm{m}, \sigma R_{j}$ é o desvio padrão dos retornos do ativo j e $\sigma R_{m}$ é o desvio padrão dos retornos do mercado.

$O$ resultado do beta é o mesmo do derivado da linha característica. Deve-se considerar o fator coeficiente de relação de forma que quanto menor o coeficiente, menor será o beta, logo menor será o risco não diversificável. (Groppelli, 1998)

A fórmula do beta proporciona dois aspectos importantes sobre 0 mesmo. O risco do ativo, que é tão grande quanto for o quociente entre o seu desvio padrão e o do mercado e que esse risco é proporcional ao coeficiente de correlação entre o ativo e o mercado. (Martelanc, 2005)

Assim, a interpretação para os possíveis resultado do beta é, conforme Martelanc (2005):

Quadro 1. Possíveis Resultados do beta, segundo Martelanc (2005).

\begin{tabular}{c|l}
\hline Beta & \multicolumn{1}{|c}{ Interpretação } \\
\hline$\beta=1,0$ & $\begin{array}{l}\mathrm{O} \text { ativo possui o mesmo risco que o } \\
\text { mercado. Isto é, se o mercado subir 5\%, o } \\
\text { ativo tenderá a subir 5\% O efeito } \\
\text { negativo se dará da mesma maneira. }\end{array}$ \\
\hline$\beta>1,0$ & $\begin{array}{l}\text { O ativo possui risco maior que o risco do } \\
\text { mercado. Isto é, se o mercado subir 5\%, o } \\
\text { ativo tenderá a subir mais que 5\%. O } \\
\text { efeito negativo se dará da mesma maneira }\end{array}$ \\
\hline$\beta<1,0$ & $\begin{array}{l}\text { O ativo possui risco menor que o risco do } \\
\text { mercado. Isto é, se o mercado subir 5\%, o } \\
\text { ativo tenderá a subir menos que 5\%. O } \\
\text { efeito negativo se dará de forma análoga. }\end{array}$ \\
\hline
\end{tabular}

Fonte: Elaborada pelo autor. 
O beta também é utilizado para a identificação da recompensa do ativo dado o seu risco. A relação entre a variação do retorno esperado de uma carteira de ativos e a variação correspondente dos possíveis betas de uma carteira é denominada quociente entre recompensa e risco, isto é, quociente recompensa/risco, também denominado de índice de Treynor em homenagem a um dos seus criadores. Este índice mede quanto de recompensa o ativo dará para cada nível de risco sistemático. (ROSS, 1997)

$$
\text { Quociente } \frac{\text { Recompensa }}{\text { Risco }}=\frac{R_{j}-R_{f}}{\beta_{j}}
$$

O quociente entre recompensa e risco deve ser sempre o mesmo para todos os ativos no mercado. Se o quociente entre recompensa e risco de um ativo for maior que o outro, todos os investidores irão demandar somente aquele ativo de maior recompensa por risco. Assim, os preços dos ativos de menor recompensa por risco cairiam e dado que as taxas de retorno e os preços variam de maneiras opostas, a recompensa por risco iria aumentar. Essas sequências de compras e vendas de ativos fariam com que, para todo ativo $i$ e $j$ : (ROSS, 1997).

$$
\frac{R_{i}-R_{f}}{\beta_{i}}=\frac{R_{j}-R_{f}}{\beta_{j}}
$$

O CAPM apresenta uma medida alternativa do risco para cada desviopadrão do ativo. É evidente que o modelo CAPM facilita a comparação das varias escalas de risco dos ativos individuais. Mas, como empregar o beta na obtenção da taxa de retorno efetiva? Para isso deverá ser usado o princípio da linha de mercado de títulos (SML). (GROPPELLI, 1998)

A linha determinada quando se representa graficamente a relação entre risco não diversificável e o retorno esperado dos mercados financeiros é denominada de linha de mercado de títulos (SML). Uma das maneiras freqüentes de se escrever a SML é considerando uma carteira que engloba todos os ativos do mercado, está carteira será denominada de carteira de mercado. (ROSS, 1997)

Sendo que todos os ativos do mercado devem estar localizados na SML, o mesmo deve ocorrer com a carteira de mercado. Para que seja determinada em que ponto da SML a carteira de mercado encontra-se, é preciso saber qual o valor do coeficiente beta $\left(\beta_{m}\right)$ da carteira de mercado. Como essa carteira representa todos os ativos do mercado, o beta deve ser igual a média, logo, $\beta=1,0$. Assim, a inclinação da SML será dada por: (ROSS, 1997)

$$
\text { Inclinação da } S M L=\frac{R_{m}-R_{f}}{\beta_{m}}=\frac{R_{m}-R_{f}}{1}=R_{m}-R_{f}
$$


A inclinação da $\mathrm{SML},\left(R_{m}-R_{f}\right)$, é considerada o prêmio por risco de uma carteira de mercado. (ROSS). O prêmio pelo risco de uma carteira de mercado é a diferença entre a taxa de retorno que se espera do mercado e a taxa de retorno dos ativos livre de riscos. Visto que o retorno do mercado é incerto, é necessário que haja um prêmio de tal forma que o investidor prefira reter um portfólio do mercado em vez de ativos sem risco, cujo retorno é praticamente conhecido. (MARTELANC, 2005)

Como, $R_{j}$ e $\beta_{j}$ são, respectivamente, o retorno esperado e o beta de qualquer ativo do mercado e consequentemente estão situados na SML. Dessa maneira, sabe-se que o seu quociente entre recompensa e risco deve ser idêntico ao do mercado, logo: (ROSS, 1997)

$$
\frac{R_{j}-R_{f}}{\beta_{j}}=R_{m}-R_{f}
$$

Assim, a taxa de retorno de um ativo será encontrada pela soma da taxa de retorno de um ativo sem risco e um prêmio pelo risco. Sendo, esse prêmio pelo risco de um ativo, definido pelo retorno médio do mercado subtraído pela taxa de retorno do ativo sem risco $\left(R_{m}-R_{f}\right)$ multiplicado pelo grau de sensibilidade do ativo, ou risco não diversificável, chamado beta $(\beta)$. Assim, dado a taxa de retorno do mercado, obtêm-se: (GROPPELLI, 1998)

$$
R_{j}=R_{f}+\beta\left(R_{m}-R_{f}\right)
$$

Essa é a versão clássica do CAPM, onde, $R_{j}$ é o retorno esperado do ativo, $R_{f}$ é o retorno do ativo sem risco, $R_{m}$ é o retorno esperado da carteira do mercado e $\beta$ é a sensibilidade do ativo em relação ao mercado. (MARTELANC, 2005)

Esse resultado, o CAPM, mostra que o retorno esperado de um ativo depende necessariamente: do valor puro do capital no tempo, quantificado pela taxa livre de risco $\left(R_{f}\right)$; do prêmio pelo risco sistemático assumido $\left(R_{m}-R_{f}\right)$, determinado pelo prêmio do risco da carteira de mercado e do grau de risco sistemático $\left(\beta_{j}\right)$, determinado pela quantidade de risco sistemático presente em um ativo. (ROSS, 1997)

As características principais do método CAPM são: a eliminação do risco não sistemático por meio da diversificação por parte do investidor e a concentração de investimentos em ativos sem risco, na carteira do mercado ou em uma combinação de ambos (MARTELANC, 2005). O CAPM é utilizado tanto para carteira de ativos quanto para ativos individuais. (ROSS, 1997)

O CAPM inicia-se com a taxa livre de risco e, então, acrescenta um prêmio pelo risco, conforme visto, e um ajuste de proporcionalidade chamado beta. A SML define a taxa de retorno de um ativo para um beta qualquer. Sempre que o retorno de um ativo for menor que sua taxa, isto quer dizer que 0 
ativo tem menor valor que o esperado, logo, este ativo está superavaliado. Quando a taxa de retorno é maior que a esperada, quer dizer então, que os ativos estão subavaliados. Essa relação apresenta a forma da linha de mercado de títulos (SML), a qual é a representação gráfica do modelo CAPM. O método da SML, por apresentar a taxa de desconto do ativo, ajuda a determinação do valor presente e a rentabilidade do ativo. (GROPPELLI, 1998)

As abordagens do CAPM e da SML fornecem alguns problemas. A existência de outros fatores, de maior importância que o mercado, na influência dos retornos esperados de um ativo torna o beta uma medida de risco não confiável. Os índices de mercado utilizados - como o Standard \& Poor's, índice Down Jones - no modelo CAPM são considerado fracos pelos acadêmicos, pois são apenas substitutos e, assim, não representam um verdadeiro índice de mercado. Outro problema está nos cálculos de valores do CAPM, acreditase que os dados utilizados são históricos, enquanto que o modelo baseia-se em valores esperados. De tal forma que, os betas históricos não podem servir de base para o estabelecimento da taxa esperado de retorno do ativo. (Groppelli, 1998)

Outros estudos, como o de Fama e French, indicam que o índice de mercado apresenta uma correlação com os retornos esperados pequena, logo, não podendo servir como índice comum para determinação do coeficiente de risco não sistemático, beta, e da SML. Isto indica que, para alguns autores, o beta e, conseqüentemente o CAPM, não são validos. Porém, em resposta às críticas apresentadas, se reitera os conceitos de CAPM como válidos, necessitando de uma pesquisa de índices comuns mais apropriadas na obtenção de betas mais confiáveis. (Groppelli, 1998)

As ideias que embasam a abordagem do CAPM apresentam apenas aproximações imperfeitas. Porém servem, ainda, como ferramenta alternativa para a determinação pelo prêmio dado pelo risco e retorno dos ativos. Necessitando apenas de descobrir uma relação de fatores que possam ser utilizadas com medida representativa comum. (Groppelli, 1998)

\section{O SEGMENTO DE COMMODITIES AGRÍCOLAS}

O texto a seguir mostra a importância das commodities agrícolas tanto no agronegócio quanto na indústria economia mundial: "O consumo de óleos vegetais tem aumentado no mundo todo, substituindo parte do consumo de gorduras animais. Embora tenham algumas especificidades no que se refere às características químicas, os óleos vegetais, mas também as gorduras animais, concorrem entre si. A maioria desses óleos são utilizados em processos industriais e na alimentação humana e animal1. Em função do aumento do consumo, a produção, que pode ser obtida através de várias espécies vegetais, também tem se elevado". (DESER -Departamento de estudos 
socioeconômicos rurais - 2002 / 2007). Daí, a importância deste segmento para a geração de emprego e renda em escala mundial.

No mercado de oleaginosas visualiza-se duas categorias. A primeira, conhecidas como oleaginosas tradicionais, tais como, soja, girassol e algodão, responsáveis pelo desempenho positivo do segmento. A segunda, conhecidos como oleaginosos potenciais, tais como, macaúba, inajá, babaçu, tucumã, pinhão - manso e dendê estão sendo pesquisadas e liberadas para o processo de produção, assim contribuindo para o aumento do desempenho do semento de oleaginosas tanto no mercado nacional como internacional.

Em relação ao Brasil o texto a seguir mostra a importância das commodities agrícolas na economia brasileira: "No Brasil, o consumo anual de óleos vegetais está em torno de 3,72 milhões de toneladas. O óleo de soja é de longe o mais consumido, chegando à 3,2 milhões de toneladas em 2006/07 ou $86 \%$ do total consumido. Em seguida, na segunda posição, aparece o óleo de algodão, com 255 mil toneladas. Considerando o óleo de palma e palmiste juntos são 195 mil toneladas consumidas em 2006/07, de acordo com o USDA, ficando na terceira posição.". (DESER -Departamento de estudos socioeconômicos rurais - 2002 / 2007). Segmento em expansão gerador de emprego e renda para a economia nacional.

O ano de 2008 manteve o padrão de resultados apresentados no período 2002/2007, ou seja, tendência de crescimento no mercado de commodities agrícolas no Brasil e no mundo, apesar da turbulência verificada no mercado internacional devido à crise estabelecida nos Estados Unidos que se espalhou pelo resto do mundo.

O mercado de commodities agrícolas em 2010 foi bastante positiva. Este mercado obteve no ano de 2010, uma recuperação em relação ao a 2009. Impulsionado pelo retorno do crescimento mundial, a produtividade do ano 2010 apresentou recuperação frente a 2009. Segundo a Conab (Companhia Nacional de Abastecimento), a produção foi estimada em 154 milhões de toneladas na safra 2010/2011, representando um acréscimo médio de $3,4 \%$ em relação à 2009/2010. No Brasil, a Conab estimou um crescimento de $2,4 \%$ na área de soja para o período 2010/2011, passando de 24,1 milhões de hectares, com produção estimada de 70 milhões de toneladas, em relação aos 23,5 milhões de hectares no ano anterior. No algodão esse crescimento aproximado foi de $5,8 \%$ e o milho também cresceu, porém em menor proporção. Em área plantada, o período de 2010/2011, correspondeu a cerca de 48 milhões de hectares, isto equivale a, aproximadamente $3 \%$ acima da área plantada no período 2009/2010.

Em relação aos preços futuros, as commodities agrícolas apresentaram, em média, crescimento. O algodão, no ano de 2010 apresentou preço futuro $64 \%$ superior ao ano anterior, enquanto o milho e a soja, no mesmo período, valorizaram $50 \%$ e $34 \%$ respectivamente. 


\section{MODELO CAPM APLICADO A EMPRESAS DO SEGMENTO DE COMMODITIES AGRÍCOLAS}

Utilizou-se o modelo CAPM, para análise de retorno de capital de duas sociedades anônimas - pertencentes ao ramo de commodities agrícolas. As firmas analisadas foram: Brasil Ecodiesel Indústria e Comercio de Biocombustíveis e Óleos Vegetais S.A. (V-Agro) e SLC Agrícola S.A., no período relativo ao ano de 2009 e 2010. O índice do mercado utilizado para esta análise foi a taxa média de crescimento do lbovespa, no mesmo período e, a taxa de retorno sem risco, foi definida pela taxa Selic, vigente nos períodos de dezembro de 2009 e dezembro de 2010.

Sendo o modelo CAPM definido por:

$$
R_{j}=R_{f}+\beta\left(R_{m}-R_{f}\right)
$$

Primeiramente, é necessário determinar a medida de volatilidade anual $(\beta)$, conhecido como coeficiente Beta, das empresas Brasil Ecodiesel Indústria e Comercio de Biocombustíveis e ÓLEOS VEGETAIS S.A. (V-AGRO) e SLC Agrícola S.A. e então definir o beta do setor. Esta medida referente ao setor será definida pela média das medidas das empresas individuais analisadas. A tabela abaixo apresenta os betas individuais das firmas e sua média, que utilizaremos como medida referente ao setor de grãos.

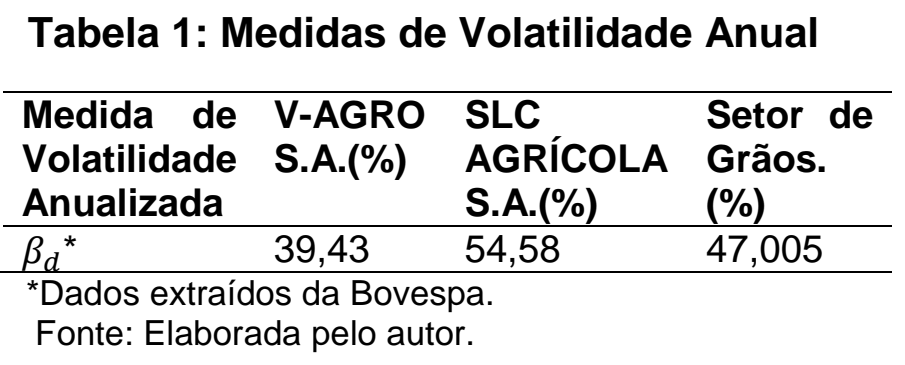

O beta da empresa, também é afetado pelo seu índice de endividamento e pelos impostos incidentes sobre o lucro. Quanto maior for o índice de endividamento, maior será o seu beta correspondente. Duas firmas idênticas, porém, com endividamentos diferentes, possuirão betas diferentes. Assim, para analisarmos as duas empresas, é necessário alavancar os betas, isto é, introduzir nas medidas de volatilidade os efeitos dos endividamentos financeiros, para podermos efetivamente compará-las. Isto é feito através da fórmula:

$$
\beta_{e}=\beta_{d}\left[1+\left(1-I_{l}\right) \frac{D}{P L}\right]
$$


Onde, $\beta_{d}$ é a medida de volatilidade desalavancada do setor, $\beta_{e}$ é o beta da empresa alavancado, $I_{l}$ é a soma das alíquotas de todos os impostos incidentes sobre o lucro - no Brasil é igual a 0,34. Por fim, $\frac{D}{P L}$ é o índice de endividamento utilizado, de forma que $D$ é o endividamento financeiro e $P L$ é o patrimônio líquido, ambos do período analisado. Assim, calculando os índices de endividamento e os betas alavancados, têm-se:

Tabela 2. Índices de endividamentos e betas alavancados.

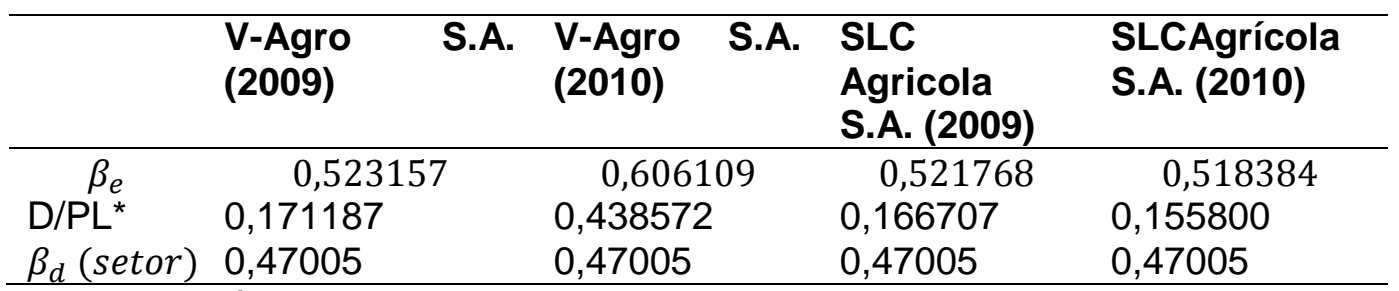

${ }^{*}$ Dados extraídos dos balanços patrimoniais 2009 e 2010 das respectivas empresas.

Fonte: Elaborada pelo autor.

Substituindo o índice de endividamento extraído dos respectivos balanços patrimoniais na expressão (1.0), definem-se os betas alavancados para o período de 2009 e 2010:

$$
\begin{aligned}
& \beta_{\text {eV-AGRO2009 }}=\beta_{d}\left[1+\left(1-I_{l}\right) \frac{D}{P L}\right]=0,47005[1+(0,66 \times 0,171187)]=0,523157 \\
& \beta_{\text {eV-AGRO2010 }}=\beta_{d}\left[1+\left(1-I_{l}\right) \frac{D}{P L}\right]=0,47005[1+(0,66 \times 0,438572)]=0,606109 \\
& \beta_{\text {eSLC2009 }}=\beta_{d}\left[1+\left(1-I_{l}\right) \frac{D}{P L}\right]=0,47005[1+(0,66 \times 0,166707)]=0,521768 \\
& \beta_{\text {eSLC2010 }}=\beta_{d}\left[1+\left(1-I_{l}\right) \frac{D}{P L}\right]=0,47005[1+(0,66 x 0,155800)]=0,518384
\end{aligned}
$$

Com os betas alavancados, pode-se agora, analisar as empresas de forma comparativa. Dessa maneira, determinam-se - através do modelo CAPM - os retornos dos ativos das empresas analisadas. Conforme dito anteriormente, utilizam-se para o índice do mercado, a taxa média de crescimento do lbovespa, no mesmo período e, a taxa de retorno sem risco foi definida pela taxa Selic, vigente nos períodos de dezembro de 2009 e dezembro de 2010. A taxa média de mercado (IBOVESPA) foi de $82,7 \% \mathrm{em}$ 2009 e 1,0\% em 2010, dados extraídos do site da Bovespa. A taxa Selic, em dezembro de 2009 era de $8,75 \%$, e em dezembro de 2010 de $10,75 \%$, dados extraídos do site do BACEN. Assim, os retornos das empresas, calculados pelo modelo CAPM, para 2009 e 2010, foram:

$$
\begin{aligned}
\mathrm{R}_{\mathrm{V}-\mathrm{AGRO2009}} & =\mathrm{R}_{\mathrm{f}}+\beta_{\mathrm{eV}-\mathrm{AGRO2009}}\left(\mathrm{R}_{\mathrm{m}}-\mathrm{R}_{\mathrm{f}}\right) \\
& =0,0875+0,523157(0,827-0,0875)=0,474374
\end{aligned}
$$




$$
\begin{gathered}
\mathrm{R}_{\mathrm{SLC2009}}=\mathrm{R}_{\mathrm{f}}+\beta_{\mathrm{eSLC} 2009}\left(\mathrm{R}_{\mathrm{m}}-\mathrm{R}_{\mathrm{f}}\right)=0,0875+0,521768(0,827-0,0875) \\
=0,473347 \\
\mathrm{R}_{\mathrm{V}-\mathrm{AGRO2010}}=\mathrm{R}_{\mathrm{f}}+\beta_{\mathrm{eV}-\mathrm{AGRO2010}}\left(\mathrm{R}_{\mathrm{m}}-\mathrm{R}_{\mathrm{f}}\right) \\
=0,1075+0,606109(0,010-0,1075)=0,048404 \\
\mathrm{R}_{\mathrm{SLC} 2010}=\mathrm{R}_{\mathrm{f}}+\beta_{\mathrm{eSLC} 2010}\left(\mathrm{R}_{\mathrm{m}}-\mathrm{R}_{\mathrm{f}}\right)=0,1075+0,518384(0,010-0,1075) \\
=0,056957
\end{gathered}
$$

Onde: $R_{V-A G R O 2009}$ é o retorno da empresa Brasil Ecodiesel Indústria $\mathrm{E}$ COMERCIO DE Biocombustíveis e ÓLEOS VEGETAIS S.A. (V-AGRO) em 2009, $R_{S L C 2009}$ é o retorno da empresa SLC AGRícOLA S.A. em 2009, $R_{V-A G R O 2010}$ é o retorno da empresa BRASIL ECODIESEL INDÚSTRIA E COMERCIO DE BIOCOMBUSTÍVEIS E ÓLEOS VEGETAIS S.A. (V-AGRO) em 2010 e $R_{S L C 2010}$ é o retorno da SLC Agrícola S.A. no período de 2010.

$O$ estudo se concentrou em duas empresas que estavam bem classificadas no banco de dados do BOVESPA\&BMF nos quesitos patrimônio e rentabilidade no ramo de negócios de commodities. Geração de empregos e renda firmes no período 2009-2010 em análise.

\section{CONSIDERAÇÕES FINAIS}

Com os resultados, verificou-se que ambas as firmas apresentaram, nos períodos analisados $\beta_{e}<1$, isto é, risco menor que o risco do mercado. Isto pode ser observado também a partir dos retornos esperados.

No ano de 2009, ano em que o mercado apresentou um alto crescimento, as firmas analisadas visualizaram retornos esperados abaixo dos retornos do mercado. Assim como em 2010, ano em que o mercado obteve uma queda na taxa de retorno, as firmas apresentaram um declínio do retorno em uma proporção menor do que o mercado, obtendo retornos esperados superiores ao mercado.

Ambos os resultados supracitados foram afetados pela oscilação presente na conjuntura econômica internacional ocorrida no mercado de agronegócios. As bolsas de mercadorias americanas e europeias refletem o desdobramento das transações no mercado de commodities agrícolas : Chicago e Frankfurt. Assim como, a bolsa de mercadorias e futuro brasileira representada pela BMF\&BOVESPA expressando o volume de transações que afetam as realizações do movimento de transações e os lucros previstos para aquelas negociações no mercado em estudo.

Foi possível observar que as duas firmas analisadas apresentaram índices de endividamento e riscos aproximadamente iguais entre si, e em relação ao mercado. Indicando assim retornos de capital muito próximo, conforme verificado nos retornos esperados. Novamente, forte influência das 
oscilações no mercado de commodities agrícolas dão o tom do resultado estabelecido.

É importante salientar que quando o cientista se debruça em estudos que leva em conta o agronegócio um elemento está em pauta : meio ambiente. Portanto, à medida que o crescimento da produção de biocombustíveis e oleaginosas acontecem é fundamental a defesa do meio ambiente através de uso de tecnologias limpas e, se possível, ao menor custo para a sociedade.

\section{REFERÊNCIAS BIBLIOGRÁFICAS}

BODIE, Z:et al. Fundamentos de Investimentos. Porto Alegre : Bookman, 2000.

GITMAN, L. J. Princípios de administração financeira. São Paulo : Pearson Prentice Hall , 2006.

GROPELLI; et al. Administração financeira. São Paulo: Editora Campus, 2005.

LENCIONE, M. A. C. Modelos de precificação. THESIS,São Paulo ano 1,vol.1,p.26-50, 2ํㅗ semestre , 2005.

MARTELANC, R; et al. Avaliação de empresas: um guia para fusões, aquisições e gestão de valor. São Paulo: Pearson Prentice Hall,2005.

ROSS, S. Probabilidade: um curso moderno com aplicações. Porto Alegre: Bookman, 2010,

ROSS,S;et al. Administração financeira. São Paulo :Editora Atlas, 1997.

SAMANEZ, C.P. Gestão de investimento e geração de valor. São Paulo: Pearson Prentice Hall,2007.

SECURATO, J. R. Calculo de tesouraria. São Paulo: Editora Saint Pauli , 2008.

SHARPE, W.F. Capital asset prices: a theory of market equilibrium under conditions of risk. The Journal of Finance, vol.19, p.425-442.

MERTON,R; BODIE,Z. Finanças. São Paulo : Editora Atlas , 2006. 